\title{
A View from the past into our collective future: the oncofertility consortium vision statement
}

Teresa K. Woodruff ${ }^{1}$ (D) - Lauren Ataman-Millhouse ${ }^{1}$ - Kelly S. Acharya ${ }^{2}$ - Teresa Almeida-Santos ${ }^{3,4,5}$. Antoinette Anazodo 6,7,8 . Richard A. Anderson ${ }^{9}$ • Leslie Appiah ${ }^{10}$. Joy Bader ${ }^{11}$. Kerri Becktell ${ }^{12}$. Robert E. Brannigan ${ }^{13}$. Lesley Breech ${ }^{14}$. Maria T. Bourlon ${ }^{15}$. Žana Bumbuliene ${ }^{16}$. Karen Burns ${ }^{17,18}$. Lisa Campo-Engelstein ${ }^{19}$. Jacira R. Campos $^{20}$. Grace M. Centola 21,22,23 . Mauricio Barbour Chehin ${ }^{24}$. Diane Chen ${ }^{25,26}$. Michel De $\operatorname{Vos}^{27,28,29}$ - Francesca E. Duncan ${ }^{1} \cdot$ Ahmed El-Damen $^{30,31} \cdot$ Douglas Fair $^{32}$. Yemi Famuyiwa ${ }^{33,34}$. Patricia Y. Fechner ${ }^{35}$ - Paula Fontoura ${ }^{36}$. Olivia Frias $^{37}$ • Sabrina A. Gerkowicz ${ }^{38,39}$. Jill Ginsberg ${ }^{40}$. Clarisa R. Gracia ${ }^{41}$ - Kara Goldman ${ }^{42}$ • Veronica Gomez-Lobo ${ }^{43}$ • Brent Hazelrigg ${ }^{11}$. Michael H. Hsieh ${ }^{44}$ • Luis R. Hoyos ${ }^{45}$ • Alfonso Hoyos-Martinez ${ }^{46}$ • Robert Jach ${ }^{47}$ • Jacek Jassem ${ }^{48}$ • Murid Javed ${ }^{49}$. Yasmin Jayasinghe ${ }^{50}$ - Roohi Jeelani ${ }^{51,52}$ • Jacqueline S. Jeruss ${ }^{53}$ - Nalini Kaul-Mahajan ${ }^{54,55}$. Jessica Keim-Malpass ${ }^{56,57}$. Tyler G. Ketterl ${ }^{58,59}$ • Mohamed Khrouf ${ }^{60}$. Dana Kimelman ${ }^{61}$ • Atsuko Kusuhara ${ }^{62}$. William H. Kutteh ${ }^{63,64}$. Monica M. Laronda ${ }^{65,66}$. Jung Ryeol Lee ${ }^{67}$ • Vicky Lehmann ${ }^{68}$. Joseph M. Letourneau ${ }^{69}$. Lynda K McGinnis $^{70}$. Eileen McMahon ${ }^{71,72}$ • Lillian R. Meacham ${ }^{73}$. Monserrat Fabiola Velez Mijangos ${ }^{74}$. $^{2}$ Molly Moravek ${ }^{75,76}$. Leena Nahata ${ }^{77}$. George Moses Ogweno ${ }^{78,79} \cdot$ Kyle E. Orwig $^{80}$ - Mary Ellen Pavone ${ }^{42}$. Fedro Alessandro Peccatori ${ }^{81}$ - Romina Ileana Pesce ${ }^{82} \cdot$ Hanna Pulaski $^{80} \cdot$ Gwendolyn Quinn $^{83} \cdot$ Ramiro Quintana $^{84}$. Tomas Quintana ${ }^{84}$ • Bruno Ramalho de Carvalho ${ }^{85}$. Rosalind Ramsey-Goldman ${ }^{86}$. Joyce Reinecke ${ }^{87}$. Fernando M. Reis ${ }^{88}$. Julie Rios ${ }^{14,37}$ - Alice S. Rhoton-Vlasak ${ }^{89} \cdot K^{9}$ nny A. Rodriguez-Wallberg ${ }^{90}$. Cassandra Roeca $^{91}$. Seth J. Rotz ${ }^{92}$ • Erin Rowell ${ }^{65,66}$. Mahmoud Salama ${ }^{1}$ • Amanda J. Saraf ${ }^{93}$ • Anibal Scarella ${ }^{94,95}$.

Tara Schafer-Kalkhoff ${ }^{96}$. Deb Schmidt ${ }^{97}$ • Suneeta Senapati ${ }^{41}$ - Divya Shah ${ }^{41}$. Ariella Shikanov ${ }^{98}$. Margarett Shnorhavorian ${ }^{99}$ • Jodi L. Skiles ${ }^{100}$ • James F. Smith ${ }^{101} \cdot$ Kristin Smith $^{42}$ • Fabio Sobral ${ }^{102}$ • Kyle Stimpert ${ }^{103}$. H. Irene Su ${ }^{104}$. Kouhei Sugimoto ${ }^{105}$. Nao Suzuki ${ }^{106}$. Mili Thakur ${ }^{107,108}$. David Victorson ${ }^{109}$. Luz Viale $^{84}$. $^{10}$

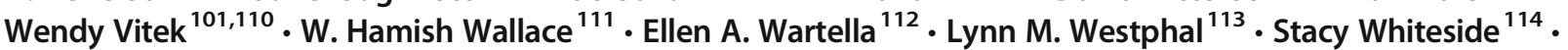
Lea H. Wilcox ${ }^{11}$. Christine Wyns ${ }^{115}$. Shuo Xiao ${ }^{116}$ - Jing Xu ${ }^{117,118}$ - Mary Zelinski ${ }^{117,118}$

Received: 21 July 2020 / Accepted: 14 October 2020 / Published online: 6 January 2021

(C) Springer Science+Business Media, LLC, part of Springer Nature 2021

\begin{abstract}
Purpose Today, male and female adult and pediatric cancer patients, individuals transitioning between gender identities, and other individuals facing health extending but fertility limiting treatments can look forward to a fertile future. This is, in part, due to the work of members associated with the Oncofertility Consortium.

Methods The Oncofertility Consortium is an international, interdisciplinary initiative originally designed to explore the urgent unmet need associated with the reproductive future of cancer survivors. As the strategies for fertility management were invented, developed or applied, the individuals for who the program offered hope, similarly expanded. As a community of practice, Consortium participants share information in an open and rapid manner to addresses the complex health care and quality-oflife issues of cancer, transgender and other patients. To ensure that the organization remains contemporary to the needs of the community, the field designed a fully inclusive mechanism for strategic planning and here present the findings of this process. Results This interprofessional network of medical specialists, scientists, and scholars in the law, medical ethics, religious studies and other disciplines associated with human interventions, explore the relationships between health, disease, survivorship, treatment, gender and reproductive longevity.
\end{abstract}

Teresa K. Woodruff

TeresaWoodruff1989@alum.northwestern.edu

Extended author information available on the last page of the article 
Conclusion The goals are to continually integrate the best science in the service of the needs of patients and build a community of care that is ready for the challenges of the field in the future.

Keywords Oncofertility $\cdot$ cancer $\cdot$ Fertility preservation

\section{Introduction}

Since the term "oncofertility" and the relatively small oncofertility consortium of research scientists and allied clinicians were organized in 2006, the group has become a robust and productive community from over 45 countries around the globe [1-12]. Since its inception, there have been a number of scientific advances, including sustained in vitro follicle growth in alginate hydrogels (with the achievement of live birth in mice, the first metaphase II (MII) egg in human, and the first nonhuman primate embryo), development of the first cryoprotectant specifically designed for and tested on non-human primate and human ovarian tissue, and the first ovarian bioprosthetics (with techniques involving decellularization, 3-D printing, and reconstituted tissue papers) and autografting of cryopreserved prepubertal rhesus testis to produce sperm and offspring [1, 13-27]. These scientific discoveries have been matched by clinical advances that include expanding options to pediatric and adolescent and young adult populations and patients with differences in sex development, transgender, and gender-diverse populations, as well as the development of optimized ovarian procurement and testicular biopsy sampling and documented methods for tissue transport [12, 28-36]. Prepubertal testicular tissue cryopreservation is now also performed in a number of centers worldwide, although this technique still remains experimental $[25,37]$. In addition, there has been a growing emphasis on the full range of patient, parent, and partner considerations including questions of legal ramifications and religious and ethical concerns [28, 38-41]. Based on the extraordinary work from groups around the world, ovarian tissue cryopreservation (OTC) is no longer designated experimental [42]. Moreover, ten US states require insurers to cover fertility preservation costs for cancer patients or any patients receiving gonadotoxic therapy. This change in the US is being mirrored in other countries and the leverage created by having a globally linked organization (Fig. 1) allows for more rapid uptake of necessary procedures $[4,43,44]$. In just over 10 years, the collective progress made by the broad and engaged stakeholders in this field has been outstanding. Now, as we approach the next 10 years of the field, our communal goal is to ensure that similar progress will be made in the future and by this coordinated effort, patients' needs will continue to be prioritized and met. As such, in early November 2019, the Oncofertility Consortium introduced a multi-month, multi-stakeholder discussion regarding the future of the field of oncofertility. Herein, we present the thoughts and conclusions of our engaged, global leadership.
Beginning in 2007, a broadly attended annual meeting has been held that created context, community, and critical mass for collaboration and increased momentum during a time frame when scientists and clinicians were working largely on their own $[45,46]$. The meeting has served as a platform for international collaboration and support in oncofertility program implementation. It has also served as a training ground and mentorship for residents, fellows, early-stage scientists, nurses, and allied health professionals from around the globe, as well as a pipeline for high school students [47-49]. The 2019 meeting had record attendance, illustrating the interest to gather and collaborate as a field. However, convening is only one dimension of our work. The community is thinking strategically about how to facilitate this meeting and how to grow the field even more broadly to advance the science and its clinical application for our patients. As such, all stakeholders were invited to participate in a strategic planning process called Vision 2030: A Roadmap for Oncofertility Research and Practices.

The strategic planning process began with an anonymized "appreciative inquiry" survey sent to a group of over 3000 oncofertility stakeholders in December 2019. Appreciative inquiry is a strength-based, positive approach to leadership development and organizational change [50]. Survey recipients included OC leadership, previous conference attendees, advocates, and other individuals who have all interacted with the OC in some way since 2007. The ultimate goal of the short, four-question appreciative inquiry was to identify the OC's core strengths and to explore how to leverage these strengths to redesign the organization to make it more effective and sustainable. Suggestions were analyzed, and a summary document was provided to the members of the leadership group for their review. Emerging themes included diversity, collaboration, networking, the annual meeting, leadership, team science, sharing, and bringing together stakeholders. (See Fig. 2 for complete appreciative inquiry survey.)

Following the appreciative inquiry, on February 7, 2020, a diverse group of leaders from the OC participated in a daylong meeting, to review the appreciative inquiry responses and discuss the resulting next steps for the field. This leadership group included clinicians and basic scientists, nurses, patient navigators, social scientists, and advocates, from the USA and abroad. Disciplines represented included oncology, urology, reproductive endocrinology and infertility, psychology, and specialists in pediatric, adolescent, and adult populations. Many of the participants serve as chairs of their respective 


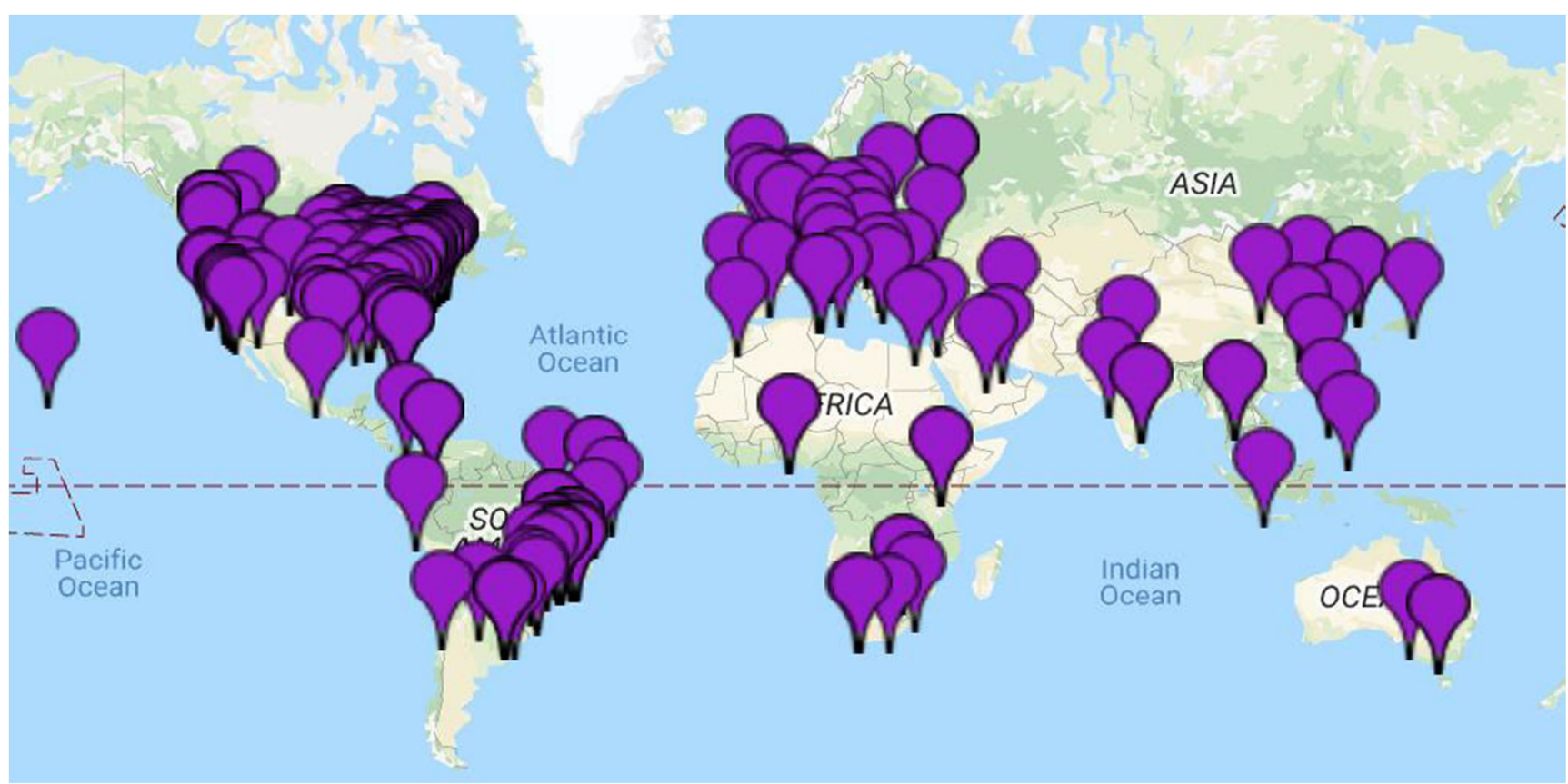

Fig. 1 Purple pins represent locations of the Oncofertility Professional Engagement Network (OPEN) member centers. OPEN consists of individuals or groups from 45 countries and is linked by basic and

\section{One
Consortium
Contity}

Oncofertility Consortium Vision 2030: Appreciative Inquiry

Thank you in advance for taking the time to complete this survey. These answers will be used to initiate the Oncofertility Consortium's Vision 2030 strategic planning process, and will help the Oncofertility Consortium construct its mission, values, and goals for the next ten years.

The goal of this survey is to leverage the Oncofertility Consortium's positive core strengths (as identified by all stakeholders) to design and redesign the organization to achieve a more effective and sustainable future.

The questions in this survey will be split in four stages:

1. Discovery

2. Dream

3. Design

4. Destiny

Please be as detailed as possible when answering open ended questions. The more details you can recall about a specific story, event, or anecdote, the better. Please focus on only the positive core strengths of the Oncofertility Consortium as the goal is to understand what we do well and how we can leverage these strengths in the future.

Question 1: Discovery: Appreciating and valuing the best of what is. What gives the Oncofertility Consortium life? What does the Oncofertility Consortium do best? What are the strengths of the Oncofertility Consortium? What do you appreciate about the Oncofertility Consortium? Include stories or information about what is working well.

Question 2: Dream: Envisioning what might be

In a blue sky environment, how do you envision the impact of the Oncofertility Consortium? Without any constraints, what results would you anticipate? What do you wish for the Oncofertility Consortium? What would be your best aspirations for the Oncofertility Consortium in five and ten years?

Question 3: Design: Determining what should be

What is the idrol should the

Oncofertility Consortium be doing? How can we build even more on the way we collaborate? Include any ideas for co-constructing this design.

Question 4: Destiny: Innovating what will be

How can the Oncofertility Consortium innovate to sustain its efforts? How can teams and individuals make the dream and design propositions a reality? How would you co-create the future of the Oncofertility Consortium?

Fig. 2 Appreciative inquiry survey clinical research a high-level of shared protocols and experiences. Member leverage work done in affiliated centers to accelerate their formation and services offered [1-7]

Oncofertility Professional Education Network (OPEN) subcommittees [1] and have exhibited exemplary leadership and engagement in the field. Together this group discussed the current state of the field, the priorities for the next 10 years, and the strategies to be used to ensure that the field is moving forward in a productive and collaborative manner. The group was also tasked with developing the OC's vision, mission, and value statements (Fig. 3), and to identify four strategic goals for the field which were (1) improve access, (2) advance research, (3) Educate, and (4) Collaborate.

\section{Improve access}

Both nationally and internationally, oncofertility professionals face common challenges. These include a lack of awareness among providers and patients, a lack of appropriate

\section{Vision Statement}

The vision of the Oncofertility Consortium is for all patients facing loss of fertility due to medical conditions and/or gonadotoxic therapies to have access to oncofertility options and services. Mission Statement

The mission of the Oncofertility Consortium is to convene the field to enable the essential conversations necessary to drive oncofertility research, clinical practice, and patient options forward. Value Statement

The Oncofertility Consortium values collegiality, multi-disciplinary collaboration, inclusiveness, innovation, and altruism. All of its values are ultimately driven by patient-centeredness.

Fig. 3 Vision, mission, and value statement issued by the oncofertility leadership team 
communication between provider and the patient-parent dyad (particularly for pediatric providers), a lack of referral pathways with no standardized models of care, cultural and religious constraints, uncertainties regarding exact risks of cancer-related infertility, insufficient funds to support oncofertility programs, and a lack of insurance coverage, and high out-of-pocket costs for patients [2, 51-53]. As such, we believe in the next 10 years, one major strategic goal of the $\mathrm{OC}$ is to improve access to care. This can be facilitated in part by increasing oncofertility awareness through the expansion of OPEN [2]. OPEN is a free, membership-based network comprised of oncofertility professionals from 45 countries. It encourages members to share resources, methodologies, and experiences to collaboratively advance the field and break down barriers to access.

First, we can continue to support new and existing centers with detailed roadmaps and patient navigation expertise to develop, re-organize, and implement oncofertility programs $[32,54]$. The OC has worked to provide centers with consent templates, protocols, business plans, and support, to facilitate efficient program implementation. The OC's network of patient navigators provides professional peer support and unprecedented opportunity for collaboration to serve both patients and programs regardless of their location. Ideally, in the future, a team of patient navigators and oncofertility experts would be equipped with standard operating procedures and guidelines and have the ability to travel to rural, remote, or new clinics and centers to raise awareness; assist with leadership engagement; and subsequently help build robust oncofertility programs. Furthermore, the resources provided by these teams would be in multiple languages, and they would be culturally adapted by local experts, who have a deeper insight into the local culture and knowledge about local resources and processes.

During the February meeting, the OC leadership team also explored the possibility of pursuing a center of excellence designation for qualified member centers. Though time intensive to procure, this designation may help enhance the depth and breadth of oncofertility services offered to patients. We discussed the several advantages to establishing a center of excellence model, including lending further credence to our work and credibility to an institution, clearly identifying highlevel institutions for patients seeking to identify a comprehensive oncofertility program at which to pursue care, as well creating a continuum of success for our centers and further incentivizing member institutions to join the OC and OPEN. We also considered, however, certain barriers to pursuing a center of excellence designation. The OC is grounded in altruism, so affixing labels of quality may be exclusionary in some instances; we would not want to disregard any centers, including those centers with limited resources. Moreover, fertility preservation in the cancer setting is extremely time sensitive. In many instances, patients may not have enough time to investigate a center's designation and respective outcome data. We would never want a center of excellence designation, or lack thereof, to prevent a patient from pursuing family building options. To achieve the full benefits of this model, proper assembly is required [55] and the leadership team will need to further examine the benefits and risks associated with this model. Ideally, as a group, we would need to determine how to create a designation that is customizable and takes into account institutional variation so that it seeks to encourage and highlight positive change and does not penalize or devalue programs that are simply resource poor.

We can also improve access by optimizing internet-based resources and by utilizing telehealth technologies to reach patients in remote locations or low-resource settings [56]. As the working world continues to evolve and patients face geographical and physical barriers to care, the utilization of telehealth would improve awareness and access to oncofertility care [57]. The transformation of clinical care through the use of telehealth due to the COVID-19 pandemic has demonstrated that timely fertility consultations can be provided remotely when patients otherwise may not have had the opportunity to be counseled on fertility risk and preservation options before beginning cancer therapy. Telehealth platforms that are Health Insurance Portability and Accountability Act (HIPAA)-compliant are being utilized to access vital healthcare services. Although many oncofertility procedures require in-person care, the initial consultations prior to treatment could be performed remotely and the patient would then be directed to the nearest clinic for treatment [58, 59]. Telehealth services provide a centralized service for initial consultation not just during trying times, but as a model for getting expertise to vulnerable or remote communities, enabling more efficient initial first steps. In the USA, the recent change in Medicare reimbursement rules to cover telemedicine consults has dramatically accelerated the financial feasibility of utilizing telemedicine for fertility preservation. Advocating for these changes to become permanent is a critical policy component for improving and maintaining access to fertility preservation expertise. Assembling a team of skilled patient navigators to conduct these remote consultations could be a priority in the field so we are able to nimbly adapt to current and future challenges.

It is important to note that we must also be mindful of the economic and geographic hindrances of oncofertility services while we explore approaches to improve access. While patients have struggled with previously reported barriers to care, specifically high out-of-pocket costs, the COVID-19 pandemic has shed light on geographic barriers to care. Although a patient can still receive timely intervention and consultation through the use of telehealth technologies, they may not be able to travel to designated centers for in-person care and procedures due to travel restrictions. The OC will continue to support translation of innovation and cutting-edge clinical 
or research advances into tangible solutions at the grassroot level. For example, in low-resource settings, member institutions can provide training to the local youth to access telemedicine technology and help set it up for patients. Similarly, member institutions can channel philanthropic resources to provide transportation for patients requiring travel to sites specializing in oncofertility procedures. The diversity of the OC membership provides us the platform to understand needs of our community and effect change at local, regional, national, and international level.

One of the steepest barriers to care remains the high cost of fertility preservation services. This is both because of the relatively high costs associated with the procedures themselves and due to the lack of insurance coverage. The OC works with other groups and professional societies, namely the Alliance for Fertility Preservation (the AFP), to advocate for improved insurance coverage. This has now been achieved in ten states, but much work remains. The OC can leverage its members' geographic diversity to form AFP "working groups" locally, to work both with their institutions and within their states toward the attainment of greater coverage. Existing template bills, sample private insurance policies, toolkits, and other materials can be shared to expedite legislative and regulatory initiatives in several states.

\section{Advance research}

Our second strategic goal is to advance basic science research since this is the backbone of all that we do. In 2007, when the OC was founded, we brought together numerous research projects with the idea that this research would eventually translate to extraordinary clinical care for cancer patients who face fertility loss. Additionally, we would have been unable to advance the field of oncofertility without establishing the current baselines of clinical need and patient outcomes. As such, it is crucial to continue the support of basic science, clinical, and translational research projects throughout the OC.

We have defined a field within science and medicine and created corridors of communication that have led to options for fertility preservation and hormone restoration in pediatric, adolescent and young adult, and adult cancer patients. We have disseminated our clinical research findings and taught our bench technologies to enable additional resources and perspectives to expand and expedite our impact. We are leading the field through team science and connected common goals and interests to enable a network of engaged professionals and trainees, both in the USA and abroad, who share a passion for oncofertility and reproductive health [60]. These teams have created a series of key intellectual and didactic products. Collectively, members of the $\mathrm{OC}$ have been involved with many of the groundbreaking discoveries that have defined our field. We reported the first restoration of reproductive function - endocrine hormone production and live birth - using engineered ovarian transplants in mice [17]. The OC has leveraged discoveries from advances in genomic medicine to improve oncofertility care by collaboration with geneticists and genetic counselors. Researchers at the frontier of bioengineering and 3-dimensional printing have enabled deployment of this exciting technology for development of the bioprosthetic ovary [61]. Moreover, we reported the first human MII eggs developed after in vitro growth of early secondary human follicles $[8,21]$.

Facilitating translational and clinical research is also a priority of the OC. Because the field is relatively young, and impact of therapy on fertility is a late outcome, it is difficult to study and, as such, comprehensive longitudinal data about oncofertility patients does not exist, but is critically needed. A number of international centers have already established registries and collaborative work with other international registries could help to identify challenges in building such large registries [62, 63]. As such, a goal of the OC would be to work with a range of investigators to collaborate on an international scale and establish a comprehensive oncofertility registry to investigate long-term outcomes in patients, such as future fertility, pregnancy outcomes, and age at menopause. This registry could track baseline data and longitudinal outcomes that can be used to guide oncofertility interventions. Though aspirational, a central data coordinating center with the capability to automatically import data from participating institutions is the ideal. Such a platform will allow participating institutions to export their data to the centralized database without the need for additional human resources, minimizing cost, and improving access for smaller institutions. Equal access to the collective, big data provided by participating institutions will allow multiple research questions to be addressed simultaneously, thus catapulting the field in key areas such as approaches to fertility preservation in vulnerable populations, long-term fertility outcomes, gynecologic and urologic late effects of cancer therapies, and barriers to referral. This international database is long overdue and critical to advancing the field.

We have been successful thus far because OC research teams have collaborated across institutions. Strengthening local, regional, and international ties and maximizing interaction among centers creates synergistic opportunities for the field. We will continue to provide the forum and platform to enable these connections through online forums and in-person conferences and training programs that expedite research discovery and we will provide support to investigators in search of research funding.

Additionally, the OC can help pursue opportunities to advocate for funding at the NIH and offer guidance for particular requests for applications (RFAs). Whether this means providing a letter of support for an NIH grant proposal or helping to identify and disseminate relevant grant and funding 
opportunities, the OC is committed to the advancement of scientific research. Furthermore, we will utilize our network to identify potential collaborators to enhance the scientific merit of proposals and grant applications. We will disseminate these research findings in journals and via social media and other outlets to broaden reach and keep the field connected through sustained engagement and an interdisciplinary approach.

\section{Educate}

The third strategic goal of the $\mathrm{OC}$ is to educate the next generation of oncofertility leaders. To date, OC leaders have trained numerous students, residents, and clinical and research fellows. Despite these continuing efforts, creating new generations of oncofertility specialists remains an unmet need. Treatment options in oncofertility are rapidly expanding, including the recent change to include ovarian tissue cryopreservation as a part of the armamentarium of established care options [42]. We expect that as increasing numbers of medical centers and healthcare providers around the world will start to offer oncofertility services, there will be a corresponding increase in the need for more oncofertility education and training across disciplines. This is especially salient for healthcare providers to ensure that fertility preservation technologies are offered safely and appropriately. Consideration should be given to formal incorporation of oncofertility into the learning objectives and content for medical school, residency, and fellowship curricula. In addition, nurses and advanced practice providers are an increasingly important and engaged cohort among the community of medical providers. Prior research among all of these groups has revealed not only oncofertility educational deficits but also a strong willingness among these cohorts to engage in oncofertility educational initiatives.

We have a rich history in education, and together, with our collaborators, we created numerous educational tools in various formats and multiple languages that have catalyzed the growth of oncofertility [1, 49, 64-66]. These items include online patient and provider resources, the first comprehensive oncofertility textbook, and educational training videos for a variety of different audiences. We also created a free online certificate course as part of the American Society for Reproductive Medicine's Air Learning platform, and an annual one-day course for trainees in a diverse array of medical specialties including reproductive endocrinology and infertility, medical and pediatric oncology, pediatric and adolescent gynecology, urology, breast oncology, rheumatology, and radiation oncology [47]. The leaders in the field have provided mentorship and hands-on training to clinicians and scientists who are interested in creating or expanding oncofertility programs. Formal pathways to connect mentees and mentors will drive expansion of this field.
One of the great traditions of the $\mathrm{OC}$ is our work with high school students in local lab-based programs complimented by the educational opportunity to participate in the annual meeting. We have combined this educational opportunity with digital materials from early childhood to adults education in order to ensure that reproductive health terms are as accessible as possible to the general public [65]. Educating the next generation of students is a core value of the $\mathrm{OC}$ and finding ways to connect students to emerging health issues while informing them about their own health are dual wins for society.

Our goal has been to have educational resources that are not only enduring but also easily updated. Educational materials and seminars have been created by a team of experts to provide common patient education materials, oncofertility program guidance, provider education materials, and core educational guidelines for specialized training in oncofertility [67-70]. Moving forward, we envision a strong continuation and broad dissemination of existing resources and the development of new repositories, didactic programs, and partnerships to provide authoritative, comprehensive educational resources to researchers, patients, and providers across pediatric and adult specialties.

\section{Collaborate}

The fourth strategic goal is to continue to collaborate and connect members of the field. Even in the earliest days of the OC, it was evident that the field would benefit greatly by including multiple disciplines and expertise. We brought together a variety of medical disciplines, all with a vested interest in the field. This included reproductive endocrinologists, oncologists, urologists, OBGYNs, allied health professionals, psychologists, nurses, embryologists, and adult and pediatric specialists. We knew ethicists and religious leaders would inevitably need to be included in the conversation. Most importantly, patients and their partners and their families were included from the beginning to ensure that all of our actions revolved around their care and ultimate well-being. Therefore, from the onset of the $\mathrm{OC}$, we proactively included them as members of the field. These cross-cutting collaborations have set the stage for ongoing partnerships, the advancement of oncofertility care, and have become a hallmark of the field.

We will continue to convene annually at the Oncofertility Conference. The OC will serve as the great facilitator of communications and collaboration among OPEN members and beyond. We will collaborate with other professional societies and regional oncofertility organizations, including, but not limited to, the International Society for Fertility Preservation (ISFP), FertiProtekt, the Japanese Society for Fertility Preservation (JSFP), the Korea Society for Fertility Preservation (KSFP), the Australasian Oncofertility Consortium, and many others [6]. We will connect with 
advocacy groups to provide support to all patients and make the critical connections to ensure their needs are being met and guarantee that the patient's perspective remains a central, guiding force of the OC. There is great value in enhancing international collaborations as we aim to gain important experiences from each other, and the OC conference will continue to serve as the venue to do so.

In addition, we must develop new ways to bring all OC communities together, especially across the oncology services and their multidisciplinary teams, with the intention of providing updated information in the field, jointly elaborating strategies for accessing options by patients and their families and, finally, reducing the resistance to the fact that fertility is an aspect of the quality of survival. Although oncofertility has gained space and prestige since its inception, as stated above, there remain concerns about the effectiveness of communication between reproductive medicine professionals and oncologists, which is evidenced by the absence of programs that offer both approaches in many clinics and hospitals. In this sense, it is also important that there is an approximation of the OC with the clinical and surgical oncology societies, which allows a joint action in the elaboration of comprehensive and broadly applicable assistance protocols.

Leadership is of paramount importance to sustain the OC for the next decade and beyond. Thus, one of the early goals of the next phase will be to conceptualize a plan for leadership and structure. Together we will develop a leadership steering committee model that involves members elected or selected from the global OC community. In addition, the leadership plan should include methods of creating and maintaining engagement, since this will positively affect every goal that the OC plans on achieving in the future.

\section{Progress made outside of the $\mathrm{OC}$}

We would be remiss if we did not discuss all of the progress made in the field outside the confines of the Oncofertility Consortium. Our colleagues in Europe specifically, many of whom are not formally part of the OC, have made groundbreaking discoveries and many of the most notable advances in oncofertility and fertility preservation. This includes live births following fertility preservation using in vitro maturation of ovarian tissue oocytes and live births reported using cryopreserved and transplanted ovarian tissue [71-75]. The progress made by these centers has been outstanding and we continue to applaud their work. We believe that if we further coalesce as a field and come together to better enable collaboration and team science, we will rapidly improve the pace of research thereby accelerating clinical breakthroughs that ultimately improve the lives of our patients.

\section{Future directions and final thoughts}

The Oncofertility Consortium was created out of the urgent unmet need of young female cancer patients with limited options for fertility management prior to receiving gonadotoxic therapies. We now have an evidence-based field of medicine that offers hope as well as real options that could only be imagined in 2006. Oncofertility has expanded from a field focused on the unmet need of young reproductive age women undergoing cancer therapy into a discipline that provides fertility preservation options for people of all ages and genders. The scope of our efforts has expanded beyond cancer to also provide options for individuals at risk of infertility due to bone marrow transplantation (BMT) for benign diseases, disorders of sexual development (DSD), autoimmune diseases, genderaffirming treatments for transgender/gender-diverse populations, and other non-oncologic conditions, including rheumatology. It has expanded beyond restoration of reproductive function to also include restoration of hormonal function alone or in combination with reproduction. The key to the success of the field is to maintain the level of cooperation and low barriers to communication between leaders and centers worldwide. The multiple publications from the global team provide evidence that the world is ready for communal work and elaborating on this opportunity is part of a successful future for the field and for the OC $[1-7,76]$. This paper outlines the strategic goals and collective priorities of our leadership; however, the implementation and tactics for achievement are up to the field. With this new strategic vision and the broad global participation in the shaping of the Oncofertility Consortium's goals, we believe the time is right for the next chapter of this organization to be written.

\section{References}

1. Smith BM, Duncan FE, Ataman L, Smith K, Quinn GP, Chang RJ, et al. The National Physicians Cooperative: transforming fertility management in the cancer setting and beyond. Future Oncol. 2018;14(29):3059-72. https://doi.org/10.2217/fon-2018-0278.

2. Salama M, Ataman-Millhouse L, Sobral F, Terrado G, Scarella A, Bourlon MT, et al. Barriers and opportunities of oncofertility practice in nine developing countries and the emerging oncofertility professional engagement network. JCO Glob Oncol. 2020;6. https://doi.org/10.1200/JGO.18.00180.

3. Salama M, Ataman L, Taha T, Azmy O, Braham M, Douik F, et al. Building oncofertility core competency in developing countries: experience from Egypt, Tunisia, Brazil, Peru, and Panama. JCO Glob Oncol. 2020;6. https://doi.org/10.1200/JGO.17.00121.

4. Rashedi AS, de Roo SF, Ataman LM, Edmonds ME, Silva AA, Scarella A, et al. Survey of fertility preservation options available to patients with cancer around the globe. J Glob Oncol. 2018;4:1-16. https://doi.org/10.1200/JGO.2016.008144.

5. Rashedi AS, de Roo SF, Ataman LM, Edmonds ME, Silva AA, Scarella A, et al. Survey of third-party parenting options associated 
with fertility preservation available to patients with cancer around the globe. J Glob Oncol. 2018;4:1-7. https://doi.org/10.1200/JGO. 2017.009944.

6. Ataman LM, Rodrigues JK, Marinho RM, Caetano JP, Chehin MB, Alves da Motta EL, et al. Creating a global community of practice for oncofertility. J Glob Oncol. 2016;2(2):83-96. https://doi.org/10. 1200/JGO.2015.000307.

7. Ataman LM, Ma Y, Duncan FE, Uzzi B, Woodruff TK. Quantifying the growth of oncofertility. Biol Reprod. 2018;99(2): 263-5. https://doi.org/10.1093/biolre/ioy068.

8. Woodruff TK. Oncofertility: a grand collaboration between reproductive medicine and oncology. Reproduction. 2015;150(3):S110. https://doi.org/10.1530/REP-15-0163.

9. Woodruff TK. From the bench to bedside to babies: translational medicine made possible by funding multidisciplinary team science. J Assist Reprod Genet. 2013;30(10):1249-53. https://doi.org/10. 1007/s10815-013-0082-2.

10. Woodruff TK. The Oncofertility Consortium-addressing fertility in young people with cancer. Nat Rev Clin Oncol. 2010;7(8):466-75. https://doi.org/10.1038/nrclinonc.2010.81.

11. Woodruff TK. The emergence of a new interdiscipline: oncofertility. Cancer Treat Res. 2007;138:3-11. https://doi.org/ 10.1007/978-0-387-72293-1_1.

12. Jeruss JS, Woodruff TK. Preservation of fertility in patients with cancer. N Engl J Med. 2009;360(9):902-11. https://doi.org/10. 1056/NEJMra0801454.

13. Xu M, Kreeger PK, Shea LD, Woodruff TK. Tissue-engineered follicles produce live, fertile offspring. Tissue Eng. 2006;12(10): 2739-46. https://doi.org/10.1089/ten.2006.12.2739.

14. Xu M, Barrett SL, West-Farrell E, Kondapalli LA, Kiesewetter SE, Shea LD, et al. In vitro grown human ovarian follicles from cancer patients support oocyte growth. Hum Reprod. 2009;24(10):253140. https://doi.org/10.1093/humrep/dep228.

15. Xu M, Banc A, Woodruff TK, Shea LD. Secondary follicle growth and oocyte maturation by culture in alginate hydrogel following cryopreservation of the ovary or individual follicles. Biotechnol Bioeng. 2009;103(2):378-86. https://doi.org/10.1002/bit.22250.

16. Xiao S, Duncan FE, Bai L, Nguyen CT, Shea LD, Woodruff TK. Size-specific follicle selection improves mouse oocyte reproductive outcomes. Reproduction. 2015;150(3):183-92. https://doi.org/10. 1530/REP-15-0175.

17. Laronda MM, Rutz AL, Xiao S, Whelan KA, Duncan FE, Roth $\mathrm{EW}$, et al. A bioprosthetic ovary created using 3D printed microporous scaffolds restores ovarian function in sterilized mice. Nat Commun. 2017;8:15261. https://doi.org/10.1038/ncomms15261.

18. Jakus AE, Laronda MM, Rashedi AS, Robinson CM, Lee C, Jordan $\mathrm{SW}$, et al. "Tissue Papers" from organ-specific decellularized extracellular matrices. Adv Funct Mater. 2017;27(3). https://doi.org/ 10.1002/adfm.201700992.

19. Laronda MM, Jakus AE, Whelan KA, Wertheim JA, Shah RN, Woodruff TK. Initiation of puberty in mice following decellularized ovary transplant. Biomaterials. 2015;50:20-9. https://doi.org/10. 1016/j.biomaterials.2015.01.051.

20. Laronda MM, Duncan FE, Hornick JE, Xu M, Pahnke JE, Whelan $\mathrm{KA}$, et al. Alginate encapsulation supports the growth and differentiation of human primordial follicles within ovarian cortical tissue. J Assist Reprod Genet. 2014;31(8):1013-28. https://doi.org/10.1007/ s10815-014-0252-X.

21. Xiao S, Zhang J, Romero MM, Smith KN, Shea LD, Woodruff TK. In vitro follicle growth supports human oocyte meiotic maturation. Sci Rep. 2015;5:17323. https://doi.org/10.1038/srep17323.

22. Lehmann V, Kutteh WH, Sparrow CK, Bjornard KL, Klosky JL. Fertility-related services in pediatric oncology across the cancer continuum: a clinic overview. Support Care Cancer. 2019;28: 3955-64. https://doi.org/10.1007/s00520-019-05248-4.
23. Xu J, Lawson MS, Yeoman RR, Pau KY, Barrett SL, Zelinski MB, et al. Secondary follicle growth and oocyte maturation during encapsulated three-dimensional culture in rhesus monkeys: effects of gonadotrophins, oxygen and fetuin. Hum Reprod. 2011;26(5): 1061-72. https://doi.org/10.1093/humrep/der049.

24. Xu J, Lawson MS, Yeoman RR, Molskness TA, Ting AY, Stouffer $\mathrm{RL}$, et al. Fibrin promotes development and function of macaque primary follicles during encapsulated three-dimensional culture. Hum Reprod. 2013;28(8):2187-200. https://doi.org/10.1093/ humrep/det093.

25. Fayomi AP, Peters K, Sukhwani M, Valli-Pulaski H, Shetty G, Meistrich ML, et al. Autologous grafting of cryopreserved prepubertal rhesus testis produces sperm and offspring. Science. 2019;363(6433):1314-9. https://doi.org/10.1126/science.aav2914.

26. Ting AY, Yeoman RR, Lawson MS, Zelinski MB. In vitro development of secondary follicles from cryopreserved rhesus macaque ovarian tissue after slow-rate freeze or vitrification. Hum Reprod. 2011;26(9):2461-72. https://doi.org/10.1093/humrep/der196.

27. Ting AY, Yeoman RR, Campos JR, Lawson MS, Mullen SF, Fahy $\mathrm{GM}$, et al. Morphological and functional preservation of pre-antral follicles after vitrification of macaque ovarian tissue in a closed system. Hum Reprod. 2013;28(5):1267-79. https://doi.org/10. 1093/humrep/det032.

28. Woodruff TK, Zoloth L, Campo-Engelstein L, Rodriguez S Oncofertility: ethical, legal, social, and medical perspectives. Preface. Cancer Treat Res. 2010;156:v-vii.

29. Dolin G, Roberts DE, Rodriguez LM, Woodruff TK. Medical hope, legal pitfalls: potential legal issues in the emerging field of oncofertility. Cancer Treat Res. 2010;156:111-34. https://doi.org/ 10.1007/978-1-4419-6518-9 9.

30. Rowell EE, Corkum KS, Lautz TB, Laronda MM, Walz AL, Madonna MB, et al. Laparoscopic unilateral oophorectomy for ovarian tissue cryopreservation in children. J Pediatr Surg. 2019;54(3):543-9. https://doi.org/10.1016/j.jpedsurg.2018.06.005.

31. Rowell EE, Corkum KS, Even KA, Laronda MM. Ovarian tissue health after laparoscopic unilateral oophorectomy: a porcine model for establishing optimized fertility preservation techniques in children. J Pediatr Surg. 2020;55:1631-8. https://doi.org/10.1016/j. jpedsurg.2019.12.014.

32. Moravek MB, Appiah LC, Anazodo A, Burns KC, Gomez-Lobo V, Hoefgen HR, et al. Development of a Pediatric Fertility Preservation Program: a report from the pediatric initiative network of the Oncofertility Consortium. J Adolesc Health. 2019;64(5): 563-73. https://doi.org/10.1016/j.jadohealth.2018.10.297.

33. Johnson EK, Finlayson C, Finney EL, Harris CJ, Tan SY, Laronda $\mathrm{MM}$, et al. Gonadal tissue cryopreservation for children with differences of sex development. Horm Res Paediatr. 2019;92(2):84 91. https://doi.org/10.1159/000502644

34. Corkum KS, Lautz TB, Johnson EK, Reimann MB, Walz AL, Lockart BA, et al. Testicular wedge biopsy for fertility preservation in children at significant risk for azoospermia after gonadotoxic therapy. J Pediatr Surg. 2019;54(9):1901-5. https://doi.org/10. 1016/j.jpedsurg.2019.01.055.

35. Corkum KS, Rhee DS, Wafford QE, Demeestere I, Dasgupta R, Baertschiger R, et al. Fertility and hormone preservation and restoration for female children and adolescents receiving gonadotoxic cancer treatments: A systematic review. J Pediatr Surg. 2019;54(11):2200-9. https://doi.org/10.1016/j.jpedsurg.2018.12. 021.

36. Duncan FE, Zelinski M, Gunn AH, Pahnke JE, O'Neill CL, Songsasen N, et al. Ovarian tissue transport to expand access to fertility preservation: from animals to clinical practice. Reproduction. 2016;152(6):R201-R10. https://doi.org/10.1530/ REP-15-0598.

37. Picton HM, Wyns C, Anderson RA, Goossens E, Jahnukainen K, Kliesch S, et al. Diseases ETFOFPIS. A European perspective on 
testicular tissue cryopreservation for fertility preservation in prepubertal and adolescent boys. Hum Reprod. 2015;30(11):2463-75. https://doi.org/10.1093/humrep/dev190.

38. Rodriguez S, Campo-Engelstein L, Emanuel L. Fertile future? Potential social implications of oncofertility. J Clin Oncol. 2013;31(6):665-7. https://doi.org/10.1200/JCO.2012.44.0990.

39. Quinn GP, Stearsman DK, Campo-Engelstein L, Murphy D. Preserving the right to future children: an ethical case analysis. Am J Bioeth. 2012;12(6):38-43. https://doi.org/10.1080/ 15265161.2012.673688.

40. Campo-Engelstein L, Chen D, Baratz AB, Johnson EK, Finlayson C. Fertility preservation for a teenager with differences (disorders) of sex development: an ethics case study. J Clin Ethics. 2019;30(2): 143-53.

41. Campo-Engelstein L, Chen D, Baratz AB, Johnson EK, Finlayson C. The ethics of fertility preservation for pediatric patients with differences (disorders) of sex development. J Endocr Soc. 2017;1(6):638-45. https://doi.org/10.1210/js.2017-00110.

42. Practice Committee of the American Society for Reproductive Medicine. Electronic address aao. Fertility preservation in patients undergoing gonadotoxic therapy or gonadectomy: a committee opinion. Fertil Steril. 2019;112(6):1022-33. https://doi.org/10. 1016/j.fertnstert.2019.09.013.

43. Rodriguez-Wallberg KA, Tanbo T, Tinkanen H, Thurin-Kjellberg A, Nedstrand E, Kitlinski ML, et al. Ovarian tissue cryopreservation and transplantation among alternatives for fertility preservation in the Nordic countries - compilation of 20 years of multicenter experience. Acta Obstet Gynecol Scand. 2016;95(9):1015-26. https:// doi.org/10.1111/aogs.12934.

44. Rodriguez-Wallberg KA, Marklund A, Lundberg F, Wikander I, Milenkovic M, Anastacio A, et al. A prospective study of women and girls undergoing fertility preservation due to oncologic and non-oncologic indications in Sweden-Trends in patients' choices and benefit of the chosen methods after long-term follow up. Acta Obstet Gynecol Scand. 2019;98(5):604-15. https://doi.org/10. 1111/aogs.13559.

45. Waimey KE, Duncan FE, Su HI, Smith K, Wallach H, Jona K, et al. Future directions in oncofertility and fertility preservation: a report from the 2011 Oncofertility Consortium conference. J Adolesc Young Adult Oncol. 2013;2(1):25-30. https://doi.org/10.1089/ jayao.2012.0035.

46. Finlayson C, Johnson EK, Chen D, Dabrowski E, Gosiengfiao Y, Campo-Engelstein L, et al. Proceedings of the working group session on fertility preservation for individuals with gender and sex diversity. Transgend Health. 2016;1(1):99-107. https://doi.org/10. 1089/trgh.2016.0008.

47. Miller EJN, Cookingham LM, Woodruff TK, Ryan GL, Summers KM, Kondapalli LA, et al. Fertility preservation training for obstetrics and gynecology fellows: a highly desired but non-standardized experience. Fertil Res Pract. 2017;3:9. https://doi.org/10.1186/ s40738-017-0036-y.

48. Faurot M, Woodruff TK. The oncofertility saturday academy: a paradigm to expand the educational opportunities and ambitions of high school girls. Cancer Treat Res. 2010;156:321-44. https:// doi.org/10.1007/978-1-4419-6518-9_25.

49. Castle M, Cleveland C, Gordon D, Jones L, Zelinski M, Winter P, et al. Reproductive science for high school students: a shared curriculum model to enhance student success. Biol Reprod. 2016;95(1):28. https://doi.org/10.1095/biolreprod.116.139998.

50. Trajkovski S, Schmied V, Vickers M, Jackson D. Using appreciative inquiry to transform health care. Contemp Nurse. 2013;45(1): 95-100. https://doi.org/10.5172/conu.2013.45.1.95.

51. Anazodo A, Laws P, Logan S, Saunders C, Travaglia J, Gerstl B, et al. How can we improve oncofertility care for patients? A systematic scoping review of current international practice and models of care. Hum Reprod Update. 2019;25(2):159-79. https://doi.org/ 10.1093/humupd/dmy038.

52. Quinn GP, Vadaparampil ST, Bell-Ellison BA, Gwede CK, Albrecht TL. Patient-physician communication barriers regarding fertility preservation among newly diagnosed cancer patients. Soc Sci Med. 2008;66(3):784-9. https://doi.org/10.1016/j.socscimed. 2007.09.013.

53. Saraf AJ, Nahata L. Fertility counseling and preservation: considerations for the pediatric endocrinologist. Transl Pediatr. 2017;6(4): 313-22. https://doi.org/10.21037/tp.2017.07.02.

54. Lautz TB, Harris CJ, Laronda MM, Erickson LL, Rowell EE. A fertility preservation toolkit for pediatric surgeons caring for children with cancer. Semin Pediatr Surg. 2019;28(6):150861. https:// doi.org/10.1016/j.sempedsurg.2019.150861.

55. Elrod JK, Fortenberry JL Jr. Centers of excellence in healthcare institutions: what they are and how to assemble them. BMC Health Serv Res. 2017;17(Suppl 1):425. https://doi.org/10.1186/ s12913-017-2340-y.

56. de Man AM, Rashedi A, Nelen W, Anazodo A, Rademaker A, de Roo S, et al. Female fertility in the cancer setting: availability and quality of online health information. Hum Fertil (Camb). 2018;23: 1-9. https://doi.org/10.1080/14647273.2018.1506891.

57. Dorsey ER, Topol EJ. State of Telehealth. N Engl J Med. 2016;375(2):154-61. https://doi.org/10.1056/NEJMra1601705.

58. Vadaparampil ST, Quinn GP. Improving communication between oncologists and reproductive specialists to promote timely referral of patients with cancer. J Oncol Pract. 2013;9(6):300-2. https://doi. org/10.1200/JOP.2013.001097.

59. Letourneau JM, Ebbel EE, Katz PP, Katz A, Ai WZ, Chien AJ, et al. Pretreatment fertility counseling and fertility preservation improve quality of life in reproductive age women with cancer. Cancer. 2012;118(6):1710-7. https://doi.org/10.1002/cncr.26459.

60. Lungeanu A, Contractor NS. The effects of diversity and network ties on innovations: The emergence of a new scientific field. Am Behav Sci. 2015;59(5):548-64. https://doi.org/10.1177/ 0002764214556804.

61. Laronda MM. Engineering a bioprosthetic ovary for fertility and hormone restoration. Theriogenology. 2020;150:8-14. https://doi. org/10.1016/j.theriogenology.2020.01.021.

62. Anazodo AC, Stern CJ, RI ML, Gerstl B, Agresta F, Cohn RJ, et al. A study protocol for the australasian oncofertility registry: monitoring referral patterns and the uptake, quality, and complications of fertility preservation strategies in Australia and New Zealand. J Adolesc Young Adult Oncol. 2016;5(3):215-25. https://doi.org/ 10.1089/jayao.2015.0062.

63. De Geyter C, Calhaz-Jorge C, Kupka MS, Wyns C, Mocanu E, Motrenko T, et al. European IVFmCftESoHR, Embryology. ART in Europe, 2015: results generated from European registries by ESHRE. Hum Reprod Open. 2020;2020(1):hoz038. https://doi. org/10.1093/hropen/hoz038.

64. Hurwitz LB, Lauricella AR, Hightower B, Sroka I, Woodruff TK, Wartella E. "When You're a Baby You Don't Have Puberty": understanding of puberty and human reproduction in late childhood and early adolescence. J Early Adolesc. 2017;37(7):925-47. https:// doi.org/10.1177/0272431616642323.

65. Smeyers C, Wallach H, Woodruff TK. Repropedia: a reproductive lexicon to fill the gap in reproductive terminology. Biol Reprod. 2012;87(4):98. https://doi.org/10.1095/biolreprod.112.104000.

66. Textbook of oncofertility research and practice: a multidisciplinary approach. 1 ed: Springer International Publishing; 2019.

67. Hand M, Kemertzis MA, Peate M, Gillam L, McCarthy M, Orme L, et al. A clinical decision support system to assist pediatric oncofertility: a short report. J Adolesc Young Adult Oncol. 2018;7(4):509-13. https://doi.org/10.1089/jayao.2018.0006.

68. Allingham C, Gillam L, McCarthy M, Zacharin M, Jayasuriya S, Heloury Y, et al. Fertility Preservation in children and adolescents 
with cancer: pilot of a decision aid for parents of children and adolescents with cancer. JMIR Pediatr Parent. 2018;1(2):e10463. https://doi.org/10.2196/10463.

69. Gardino SL, Jeruss JS, Woodruff TK. Using decision trees to enhance interdisciplinary team work: the case of oncofertility. J Assist Reprod Genet. 2010;27(5):227-31. https://doi.org/10.1007/ s10815-010-9413-8.

70. Bortoletto P, Confino R, Smith BM, Woodruff TK, Pavone ME. Practices and attitudes regarding women undergoing fertility preservation: a survey of the National Physicians Cooperative. J Adolesc Young Adult Oncol. 2017;6(3):444-9. https://doi.org/10. 1089/jayao.2017.0016.

71. Segers I, Bardhi E, Mateizel I, Van Moer E, Schots R, Verheyen G, et al. Live births following fertility preservation using in-vitro maturation of ovarian tissue oocytes. Hum Reprod. 2020;35(9):202636. https://doi.org/10.1093/humrep/deaa175.

72. Donnez J, Dolmans MM. Ovarian cortex transplantation: 60 reported live births brings the success and worldwide expansion of the technique towards routine clinical practice. J Assist Reprod Genet. 2015;32(8):1167-70. https://doi.org/10.1007/s10815-015-0544-9.
73. Donnez J, Silber S, Andersen CY, Demeestere I, Piver P, Meirow $\mathrm{D}$, et al. Children born after autotransplantation of cryopreserved ovarian tissue. a review of 13 live births. Ann Med. 2011;43(6): 437-50. https://doi.org/10.3109/07853890.2010.546807.

74. Dittrich R, Hackl J, Lotz L, Hoffmann I, Beckmann MW. Pregnancies and live births after 20 transplantations of cryopreserved ovarian tissue in a single center. Fertil Steril. 2015;103(2): 462-8. https://doi.org/10.1016/j.fertnstert.2014.10.045.

75. Demeestere I, Simon P, Dedeken L, Moffa F, Tsepelidis S, Brachet $\mathrm{C}$, et al. Live birth after autograft of ovarian tissue cryopreserved during childhood. Hum Reprod. 2015;30(9):2107-9. https://doi. org/10.1093/humrep/dev128.

76. Bourlon MT, Anazodo A, Woodruff TK, Segelov E. Oncofertility as a Universal Right and a Global Oncology Priority. JCO Glob Oncol. 2020;6:314-6. https://doi.org/10.1200/GO.19.00337.

Publisher's note Springer Nature remains neutral with regard to jurisdictional claims in published maps and institutional affiliations.

\section{Affiliations}

Teresa K. Woodruff ${ }^{1}$ (1) $\cdot$ Lauren Ataman-Millhouse ${ }^{1} \cdot$ Kelly S. Acharya $^{2} \cdot$ Teresa Almeida-Santos $^{3,4,5}$. Antoinette Anazodo ${ }^{6,7,8} \cdot$ Richard A. Anderson $^{9}$. Leslie Appiah ${ }^{10}$. Joy Bader ${ }^{11}$ - Kerri Becktell ${ }^{12}$. Robert E. Brannigan ${ }^{13} \cdot$ Lesley Breech $^{14} \cdot$ Maria T. Bourlon $^{15} \cdot$ Žana Bumbuliene ${ }^{16} \cdot$ Karen Burns $^{17,18}$. Lisa Campo-Engelstein ${ }^{19}$ • Jacira R. Campos ${ }^{20}$ • Grace M. Centola ${ }^{21,22,23}$ • Mauricio Barbour Chehin ${ }^{24}$. Diane Chen ${ }^{25,26}$. Michel De Vos ${ }^{27,28,29}$ • Francesca E. Duncan ${ }^{1}$ - Ahmed El-Damen ${ }^{30,31}$ - Douglas Fair ${ }^{32}$ - Yemi Famuyiwa ${ }^{33,34}$. Patricia Y. Fechner ${ }^{35}$ • Paula Fontoura ${ }^{36}$. Olivia Frias ${ }^{37}$ - Sabrina A. Gerkowicz ${ }^{38,39}$ • Jill Ginsberg ${ }^{40}$. Clarisa R. Gracia ${ }^{41}$. Kara Goldman ${ }^{42}$ • Veronica Gomez-Lobo ${ }^{43}$ - Brent Hazelrigg ${ }^{11}$ • Michael H. Hsieh ${ }^{44}$ • Luis R. Hoyos ${ }^{45}$. Alfonso Hoyos-Martinez ${ }^{46} \cdot$ Robert Jach $^{47}$. Jacek Jassem ${ }^{48} \cdot$ Murid Javed $^{49} \cdot$ Yasmin Jayasinghe $^{50}$. Roohi Jeelani ${ }^{51,52}$ • Jacqueline S. Jeruss ${ }^{53}$ • Nalini Kaul-Mahajan ${ }^{54,55}$. Jessica Keim-Malpass ${ }^{56,57}$. Tyler G. Ketterl ${ }^{58,59}$. Mohamed Khrouf $^{60}$ • Dana Kimelman ${ }^{61}$ • Atsuko Kusuhara ${ }^{62}$ • William H. Kutteh ${ }^{63,64}$ • Monica M. Laronda ${ }^{65,66}$. Jung Ryeol Lee ${ }^{67}$. Vicky Lehmann ${ }^{68}$. Joseph M. Letourneau ${ }^{69} \cdot$ Lynda K McGinnis $^{70}$ • Eileen McMahon ${ }^{71,72}$. Lillian R. Meacham ${ }^{73}$. Monserrat Fabiola Velez Mijangos ${ }^{74}$ Molly Moravek $^{75,76}$ - Leena Nahata ${ }^{77}$. George Moses Ogweno ${ }^{78,79}$. Kyle E. Orwig ${ }^{80}$ - Mary Ellen Pavone ${ }^{42}$. Fedro Alessandro Peccatori ${ }^{81}$. Romina lleana Pesce ${ }^{82} \cdot$ Hanna Pulaski $^{80} \cdot$ Gwendolyn Quinn $^{83} \cdot$ Ramiro Quintana $^{84} \cdot$ Tomas Quintana $^{84}$. Bruno Ramalho de Carvalho ${ }^{85}$. Rosalind Ramsey-Goldman ${ }^{86}$ • Joyce Reinecke ${ }^{87}$. Fernando M. Reis ${ }^{88}$. Julie Rios ${ }^{14,37}$. Alice S. Rhoton-Vlasak ${ }^{89}$ - Kenny A. Rodriguez-Wallberg ${ }^{90}$. Cassandra Roeca ${ }^{91}$ • Seth J. Rotz ${ }^{92}$ - Erin Rowell ${ }^{65,66}$. Mahmoud Salama ${ }^{1}$. Amanda J. Saraf ${ }^{93}$. Anibal Scarella ${ }^{94,95}$ • Tara Schafer-Kalkhoff ${ }^{96}$ • Deb Schmidt ${ }^{97}$. Suneeta Senapati ${ }^{41}$. Divya Shah ${ }^{41}$. Ariella Shikanov ${ }^{98}$ - Margarett Shnorhavorian ${ }^{99}$. Jodi L. Skiles ${ }^{100}$.

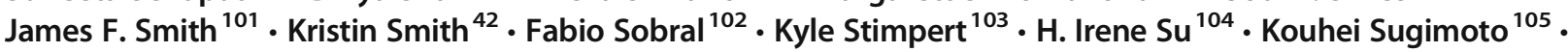
Nao Suzuki ${ }^{106}$. Mili Thakur ${ }^{107,108} \cdot$ David Victorson $^{109} \cdot$ Luz Viale $^{84} \cdot$ Wendy Vitek $^{101,110} \cdot$ W. Hamish Wallace ${ }^{111}$. Ellen A. Wartella ${ }^{112}$. Lynn M. Westphal ${ }^{113}$. Stacy Whiteside ${ }^{114}$ • Lea H. Wilcox ${ }^{11}$. Christine Wyns ${ }^{115}$. Shuo Xiao ${ }^{116}$. Jing $\mathrm{Xu}^{117,118} \cdot$ Mary Zelinski ${ }^{117,118}$

1 Department of Obstetrics and Gynecology, Feinberg School of Medicine, Northwestern University, Chicago, IL, USA

2 Department of Obstetrics and Gynecology, Division of Reproductive Endocrinology, Duke Fertility Center, Durham, NC, USA

3 Reproductive Medicine Unit, Coimbra Hospital and University Centre, Coimbra, Portugal

4 Clinical Academic Center of Coimbra, Coimbra, Portugal

5 Faculty of Medicine, University of Coimbra, Coimbra, Portugal
6 Kids Cancer Centre, Sydney Children's Hospital, Nelune Comprehensive Cancer Centre, Sydney, Australia

7 Prince of Wales Hospital, Sydney, Australia

8 School of Women's and Children's Health, University of New South Wales, Sydney, Australia

9 Centre for Reproductive Health, University of Edinburgh, Edinburgh, Scotland, UK 
10 Department of Obstetrics and Gynecology, The University of Colorado School of Medicine, Aurora, CO, USA

11 ReproTech, Ltd., Saint Paul, MN, USA

12 Medical College of Wisconsin, Milwaukee, WI, USA

13 Department of Urology, Feinberg School of Medicine, Northwestern University, Chicago, IL, USA

14 Department of Surgery, Cincinnati Children's Hospital Medical Center, Cincinnati, OH, USA

15 Hemato-Oncology Department, Instituto Nacional de Ciencias Médicas y Nutrición Salvador Zubirán, Mexico City, Mexico

16 Clinic of Obstetrics and Gynecology, Institute of Clinical Medicine, Faculty of Medicine, Vilnius University, Vilnius, Lithuania

17 Department of Pediatrics, University of Cincinnati College of Medicine, Cincinnati, OH, USA

18 Division of Oncology, Cancer and Blood Diseases Institute, Cincinnati Children's Hospital Medical Center, Cincinnati, OH, USA

19 Institute for the Medical Humanities, Department of Preventive Medicine and Population Health, University of Texas Medical Branch, Galveston, TX, USA

20 Lab For Life Human Reproduction Center, Sao Paulo, Brazil

21 Dadi, Inc., Brooklyn, NY, USA

22 Phoenix Sperm Bank of Seattle Sperm Bank, Phoenix, AZ, USA

23 New England Cryogenic Center/New England Cord Blood Bank, Marlborough, MA, USA

24 Huntington Reproductive Medicine, Sao Paulo, SP, Brazil

25 Potocsnak Family Division of Adolescent and Young Adult Medicine and Pritzker Department of Psychiatry and Behavioral Health, Ann \& Robert H. Lurie Children's Hospital of Chicago, Chicago, IL, USA

26 Departments of Psychiatry and Behavioral Sciences, and Pediatrics, Northwestern University Feinberg School of Medicine, Chicago, IL, USA

27 Centre for Reproductive Medicine, UZ Brussel, Brussels, Belgium

28 Follicle Biology Laboratory (FOBI), Vrije Universiteit Brussel, Brussels, Belgium

29 Department of Obstetrics, Gynecology, Perinatology and Reproductology, Institute of Professional Education, Sechenov University, Moscow, Russia

30 IVIRMA Middle East Fertility Clinic, Abu Dhabi, United Arab Emirates

31 Division of Embryology and Comparative Anatomy, Faculty of Science, Cairo University, Giza, Egypt

32 Department of Pediatrics, Division of Pediatric Hematology/ Oncology, University of Utah, Primary Children's Hospital, Huntsman Cancer Institute, Salt Lake City, UT, USA
33 Montgomery Fertility Center, Rockville, MD, USA

34 Department of Obstetrics and Gynecology, George Washington University School of Medicine, Washington, DC, USA

35 Department of Pediatrics, Division of Endocrinology, Seattle Children's Hospital, University of Washington, Seattle, WA, USA

36 Rio de Janeiro Sperm Bank, Rio de Janeiro, Brazil

37 Department of Obstetrics and Gynecology, College of Medicine, University of Cincinnati, Cincinnati, OH, USA

38 IVFMD, Boca Raton, FL, USA

39 IVFMD, Cooper City, FL, USA

40 Department of Pediatrics, Perelman School of Medicine, University of Pennsylvania, Philadelphia, PA, USA

41 Division of Reproductive Endocrinology \& Infertility, University of Pennsylvania, Philadelphia, PA, USA

42 Department of Obstetrics and Gynecology, Reproductive Endocrinology and Infertility, Northwestern University Feinberg School of Medicine, Chicago, IL, USA

43 Eunice Kennedy Shriver National Institute of Child Health and Human Development, National Institutes of Health, Bethesda, MD, USA

44 Department of Urology, George Washington University, Washington, DC, USA

45 Division of Reproductive Endocrinology and Infertility, Department of Obstetrics and Gynecology, University of California, Los Angeles, CA, USA

46 Department of Pediatrics, Section of Pediatric Diabetes and Endocrinology, Baylor College of Medicine, Texas Children's Hospital, Houston, TX, USA

47 Department of Obstetrics and Gynecology, Medical College Jagiellonian University, Krakow, Poland

48 Department of Oncology and Radiotherapy, Medical University of Gdansk, Gdansk, Poland

49 OriginElle Fertility Clinic and Women's Health Centre, Ottawa, ON, Canada

50 Department of Obstetrics \& Gynaecology Royal Women's Hospital, University of Melbourne, Royal Children's Hospital, Melbourne, Australia

51 Vios Fertility Institute, Chicago, IL, USA

52 Department of Obstetrics and Gynecology, Wayne State School of Medicine, Detroit, MI, USA

53 Departments of Surgery, Pathology, and Biomedical Engineering, University of Michigan, Ann Arbor, MI, USA

54 Mother \& Child Hospital, New Delhi, India

55 Ferticity Fertility Clinics, New Delhi, India 
56 School of Nursing, University of Virginia, Charlottesville, VA, USA

57 Department of Pediatrics, School of Medicine, University of Virginia, Charlottesville, VA, USA

58 Department of Pediatrics, Division of Pediatric Hematology/ Oncology, University of Washington, Seattle, WA, USA

59 Cancer and Blood Disorders Center, Seattle Children's Hospital, Seattle, WA, USA

60 FERTILLIA, Clinique la Rose, Tunis, Tunisia

61 Centro de Esterilidad Montevideo, Montevideo, Uruguay

62 Department of Obstetrics and Gynecology, The Jikei University School of Medicine, Tokyo, Japan

63 Department of Reproductive Endocrinology, Vanderbilt University School of Medicine, Nashville, TN, USA

64 Department of Surgery, St. Jude Children's Research Hospital, Memphis, TN, USA

65 Stanley Manne Children's Research Institute, Ann \& Robert H. Lurie Children's Hospital of Chicago, Chicago, IL, USA

66 Department of Pediatrics, Feinberg School of Medicine, Northwestern University, Chicago, IL, USA

67 Division of Reproductive Endocrinology, Department of Obstetrics and Gynecology, Seoul National University College of Medicine, Seoul National University Bundang Hospital, Fertility Preservation and Enhancement Research Laboratory, Seongnam, Korea

68 Department of Medical Psychology, Amsterdam University Medical Centers, Cancer Center Amsterdam, Amsterdam, The Netherlands

69 University of Utah Center for Reproductive Medicine, Salt Lake City, UT, USA

70 Department of Obstetrics and Gynecology, University of Southern California, Los Angeles, CA, USA

71 Sinai Health System, Mount Sinai Fertility, Toronto, Ontario, Canada

72 University of Toronto, Toronto, Ontario, Canada

73 Department of Pediatrics, Aflac Cancer Center of Children's Healthcare of Atlanta, Emory University, Atlanta, GA, USA

74 Biology of Human Reproduction Department, Instituto Nacional de Ciencias Médicas y Nutrición Salvador Zubirán, Mexico City, Mexico

75 Department of Obstetrics and Gynecology, University of Michigan, Ann Arbor, MI, USA

76 Department of Urology, University of Michigan, Ann Arbor, MI, USA

77 Department of Pediatrics, The Ohio State University College of Medicine, Columbus, OH, USA
78 Reproductive Endocrinology and Fertility, Department of Obstetrics and Gynecology, The Nairobi Hospital, Nairobi, Kenya

79 Esis Health Services (EHS), Nairobi, Kenya

80 Department of Obstetrics, Gynecology and Reproductive Sciences, Magee-Womens Research Institute, University of Pittsburgh School of Medicine, Pittsburgh, PA, USA

81 Fertility \& Procreation Unit, Division of Gynecologic Oncology, European Institute of Oncology IRCCS, Milan, Italy

82 Reproductive Medicine Unit, Obstetrics and Gynecology Department, Hospital Italiano de Buenos Aires, Buenos Aires, Argentina

83 Departments of Obstetrics and Gynecology, Center for Medical Ethics, Population Health, Grossman School of Medicine, New York University, New York, NY, USA Procrearte, Buenos Aires, Argentina

85 Hospital Sírio-Libanês Brasília, Brasília, DF, Brazil

86 Department of Medicine/Division of Rheumatology, Feinberg School of Medicine, Northwestern University, Chicago, IL, USA

87 Alliance for Fertility Preservation, Lafayette, CA, USA

88 Universidade Federal de Minas Gerais, Belo Horizonte, Brazil

89 Department of Obstetrics and Gynecology, University of Florida College of Medicine, Gainesville, FL, USA

90 Department of Reproductive Medicine, Karolinska University Hospital and Karolinska Institutet, Stockholm, Sweden

91 Division of Reproductive Endocrinology \& Infertility, Department of Obstetrics \& Gynecology, University of Colorado School of Medicine, Aurora, CO, USA

92 Department of Pediatric Hematology, Oncology, and Blood and Marrow Transplantation, Cleveland Clinic Children's Hospital, Cleveland, OH, USA

93 Riley Hospital for Children at Indiana University, Indianapolis, IN, USA

94 Centro de Reproducción Humana, Facultad Medicina, Universidad de Valparaíso, Valparaíso, Chile

95 Departamento de Obstetricia y Ginecología, Escuela de Medicina, Universidad de Valparaíso, Valparaíso, Chile

96 Cincinnati Children's Hospital Medical Center, Cincinnati, OH, USA

97 Children's Hospital of Wisconsin, Milwaukee, WI, USA

98 Department of Biomedical Engineering, Department of Obstetrics and Gynecology, University of Michigan, Ann Arbor, MI, USA

99 Department of Urology, Division of Pediatric Urology, Seattle Children's Hospital, Seattle, University of Washington, Seattle, WA, USA

100 Department of Pediatrics, Indiana University School of Medicine, Indianapolis, IN, USA 
101 Department of Urology, University of California, San Francisco, San Francisco, CA, USA

102

Department of Hematology/Oncology, University Hospitals Cleveland Medical Center, Cleveland, OH, USA

104

Department of Obstetrics, Gynecology and Reproductive Sciences, University of California, San Diego, CA, USA

105

International Center for Reproductive Medicine, Dokkyo Medical University, Saitama Medical Center, Saitama, Japan

Department of Obstetrics and Gynecology, St. Marianna University School of Medicine, Kawasaki, Japan

Department of Medical Social Sciences, Feinberg School of Medicine, Northwestern University, Chicago, IL, USA
Paediatric Oncology, University of Edinburgh \& Royal Hospital for Sick Children, Edinburgh, Scotland, UK

112 Center on Media and Human Development, School of Communication, Northwestern University, Evanston, IL, USA

Department of Obstetrics and Gynecology, Stanford University School of Medicine, Palo Alto, CA, USA

114 Fertility \& Reproductive Health Program, Department of Hematology/Oncology/BMT, Nationwide Children's Hospital, Columbus, OH, USA

115 Cliniques universitaires Saint-Luc, Université Catholique de Louvain, Brussels, Belgium

116 Department of Pharmacology and Toxicology, Ernest Mario School of Pharmacy, Environmental Health Sciences Institute, Rutgers University, New Brunswick, NJ, USA

117 Division of Reproductive \& Developmental Sciences, Oregon National Primate Research Center, Oregon Health \& Science University, Beaverton, OR, USA

Department of Obstetrics \& Gynecology, School of Medicine, Oregon Health \& Science University, Portland, OR, USA 